\title{
Caractéristiques de la prise d'eau à Andekalela pour l'exclusion des sédiments
}

\author{
The Andekalela water intake characteristics \\ for excluding sediments
}

\section{F.E. Parkinson}

\author{
Laboratoire d'Hydraulique \\ La Salle Ltée \\ La Salle, Québec, Canada
}

\author{
J.L. Gordon
}

Les Consultants

Monenco Ltée

Montréal, Québec, Canada
B. Rakotondrafara

JIRAMA

Antananarivo

Madagascar

Le projet hydroélectrique Andekaleka sur la Rivière Vohitra à Madagascar (chute : $214 m ; 56 \mathrm{MW}$ ) a été mis en service en 1982. L'aménagement comprend une prise d'eau conçue pour que les sédiments de la rivière ne puissent pas s'introduire dans la galerie d'amenée. Des restrictions topographiques du site imposaient une conception ne permettant qu'un accroissement faible du niveau de l'eau en amont; elles ne permettaient pas non plus l'aménagement de bassins de sédimentation classiques. A la suite des études sur modèle hydraulique, la conception préliminaire, a été modifiée de façon à inclure une prise d'eau à tube-collecteur ainsi que deux passes pour l'évacuation des sédiments et des crues, munies de vannes secteurs. Après deux ans d'exploitation, les résultats des relevés ont confirmé les résultats des essais sur modèle (conditions d'écoulement, concentration en sédiments).

The Andekaleka hydroelectric project on the Vohitra River in Madagascar (head: $214 \mathrm{~m}, 56 \mathrm{Mw}$ ) began operation in 1982. The works include an intake designed to exclude bedload from entering the water passages. Topographic restrictions at the site required a design with only a nominal increase in headwater level, and did not permit the use of normal sediment settling basins. Scale hydraulic model studies suggested modifications to the preliminary design, which were developed around a collecting-tube intake integrated into the overflow spillway structure. A submerged concrete wall divides the head-pond into two parts, and diverts the bedload toward two large spillway sector gates for clearing during flood flows. Field inspections after two years operation confirmed the model forecasts in termes of water flow and sediment transport.

Le projet hydroélectrique Andekaleka est situé dans la partie centrale-est de Madagascar où une chute nette de $214 \mathrm{~m}$ a été aménagée pour produire $56 \mathrm{MW}$ de puissance dans une centrale souterraine, prévue avec un espace suffisant pour permettre d'en doubler la capacité. On y a produit de l'électricité pour la première fois en avril 1982 après trois ans de travaux.

Ce complexe énergétique comprend notamment un bief amont et une prise d'eau inusités, conçus pour exclure les sédiments charriés par la rivière, qui font l'objet de cette communication. Une description détaillée du projet a été publiée ailleurs (1). L'implantation générale du barrage est illustrée à la figure 1. Plusieurs conceptions différentes de la prise d'eau ont été mises à l'essai sur un modèle hydraulique à lit mobile jusqu'à ce que l'agencement satisfaisant ait été obtenu.

Une inspection du projet à la fin de la deuxième année d'exploitation n'a révélé qu'un volume négligeable de dépôts dans la galerie d'amenée confirmant les résultats des essais sur modèle. 




Figure 1. - Implantation générale.

Le complexe est situé sur la rivière Vohitra où une chute brute de $238 \mathrm{~m}$ a été aménagée entre la prise d'eau et la sortie de la galerie de restitution. En plus de la prise d'eau, le projet comprend :

- une galerie de $2130 \mathrm{~m}$ de long menant à la rivière Sihantsiva.

- une traverse aérienne de $70 \mathrm{~m}$ de long au-dessus de la rivière.

- une galerie de $1800 \mathrm{~m}$ de long jusqu'à la cheminée d'équilibre.

- une cheminée d'équilibre et une conduite forcée verticale.

- une conduite forcée horizontale de haute charge de $220 \mathrm{~m}$ de long.

- une centrale dotée de deux unités de $28 \mathrm{MW}$ et l'espace nécessaire pour deux autres.

- une chambre d'équilibre aval.

- une galerie de restitution de $600 \mathrm{~m}$ de long.

La traverse aérienne comprend une section amovible permettant l'accès aux galeries non blindées à basse pression pour des réparations majeures. Immédiatement en amont du pont, il y a un piège à sédiments qui communique avec un tuyau de vidange muni d'une vanne de réglage pour effectuer des chasses de sédiments.

\section{Le problème de dérivation}

Aucune mesure du débit de charriage n'était disponible, mais une visite au site a permis d'identifier la granulométrie de particules à partir de laquelle une valeur annuelle de 290000 tonnes a été calculée. Quelques échantillons pris lors de relevés antérieurs permettaient d'évaluer la charge en suspension à 140000 tonnes par an.
Il y a de plus des débris flottants tels les enveloppes de riz, feuilles, herbes et petites branches jusqu'à des troncs d'arbres et des bambous, provenant des fermes et des berges en amont.

Il n'y a actuellement aucun réglage du débit de la rivière à part le petit réservoir créé au site. Le débit de la rivière est suffisant pour les deux premières unités, mais il devra être accru à l'aide d'un réservoir à Ankorahotra, une fois la centrale terminée. La crue de 1000 ans, estimée à $7000 \mathrm{~m}^{3} / \mathrm{sec}$. a été retenue pour l'étude de l'évacuateur de crues. Le débit minimum est de $16 \mathrm{~m}^{3} / \mathrm{sec}$.

La rivière ne présente pas de concentrations de chute où un barrage pourrait être construit avec de l'espace en aval pour des pièges à sédiments. Les sites du barrage de dérivation et de sortie de canal de fuite ont donc été choisis en vue d'obtenir la plus haute chute, avec les tunnels les plus courts.

Le site retenu pour le barrage de dérivation se trouve à une fourche de la rivière où il y aurait assez d'espace pour permettre des dérivations temporaires ainsi que les manœuvres de l'équipement de construction. La pente de la rivière en amont est relativement faible et elle assure une petite retenue ayant un volume de stockage de 120000 $\mathrm{m}^{3}$. Ce site présente cependant les désavantages suivants :

- un niveau d'eau aval élevé qui réduit la chute hydraulique, en période de crue, de part et d'autre du barrage de dérivation à environ $1 \mathrm{~m}$.

- une voie ferrée longeant la rive droite de la rivière qui limite la hauteur du barrage.

L'ingénieur a donc dû faire face au problème de mise au point d'un concept qui :

- éviterait que les matériaux charriés par la rivière entrent dans la prise d'eau.

- comprendrait un ouvrage qui ne remonte pas le niveau 
de crue de plus d'un mètre au-dessus du niveau de crue naturel.

- assurerait un moyen de passage en aval des sédiments charriés par la rivière.

- comprendrait un ouvrage qui n'exige pas de dessableur.

Ceci s'avéra une tâche difficile qui exigeait l'utilisation d'un modèle hydraulique sur lequel cinq différentes prises d'eau ont été essayées avant de parvenir à un concept respectant toutes les exigences.

\section{Le concept d'origine}

Le concept mis sur pied dans le rapport d'ingénierie prévoyait un barrage de dérivation en travers de la rivière avec deux vidanges de fond dont une de $2,66, \mathrm{~m} \times 3,66 \mathrm{~m}$ de large, adjacente au contrefort gauche, ef l'autre plus petite, de $0,9 \mathrm{~m} \times 1,5 \mathrm{~m}$ de large traversant le déversoir à droite juste en aval de la tour de la prise d'eau. Le fond des grilles à débris de la prise d'eau a été calé à $1,5 \mathrm{~m}$ en-dessous du seuil du pertuis de dévasement. Un mur central submergé devait protéger la prise d'eau des sédiments, tel qu'indiqué à la figure 2.

Les essais du modèle hydraulique ont démontré que ce concept ne convenait pas puisque les dépôts passaient au-dessus du mur central pour entrer directement dans la prise d'eau.

La vidange de fond du côté gauche était trop petite pour attirer les matériaux charriés le long du thalweg, vers la sortie, et le pertuis de droite était trop petit et trop loin en aval pour éloigner les sédiments de la prise d'eau. La progression des dépôts est illustrée à la figure 3. A la fin de l'essai, les solides avaient rempli la dépression devant la prise d'eau et entraient dans celle-ci.

Une analyse des lacunes du concept d'origine indiqua que tout agencement modifié devrait comprendre les éléments suivants :

- une passe de grand débit dans le chenal profond principal à gauche.

- un mur submergé afin de détourner les matériaux charriés vers la passe, en même temps créant le bassin de prise contre la rive droite.

- un niveau du seuil de la prise d'eau bien au-dessus du niveau du seuil de la passe.

- implantation de la prise d'eau aussi près que possible de la passe.

- l'incorporation d'un pont d'accès pour faciliter les opérations et l'entretien.

La remontée du niveau du seuil de la prise d'eau a posé un sérieux problème puisqu'il n'y avait qu'une distance verticale relativement faible entre le niveau du seuil de la passe (élév. 350 ) et le niveau minimum de la retenue (élév. 356). De plus, on croyait qu'à l'élévation 350 le seuil de la passe serait trop profond et que son rendement serait affecté par un niveau d'eau aval élevé. Il fut donc décidé de changer la forme de la prise d'eau à un concept comprenant un tube-collecteur avec grilles à débris calées juste sous le niveau minimum de la retenue et s'étendant vers le bas jusqu'à une élévation d'environ 354 .

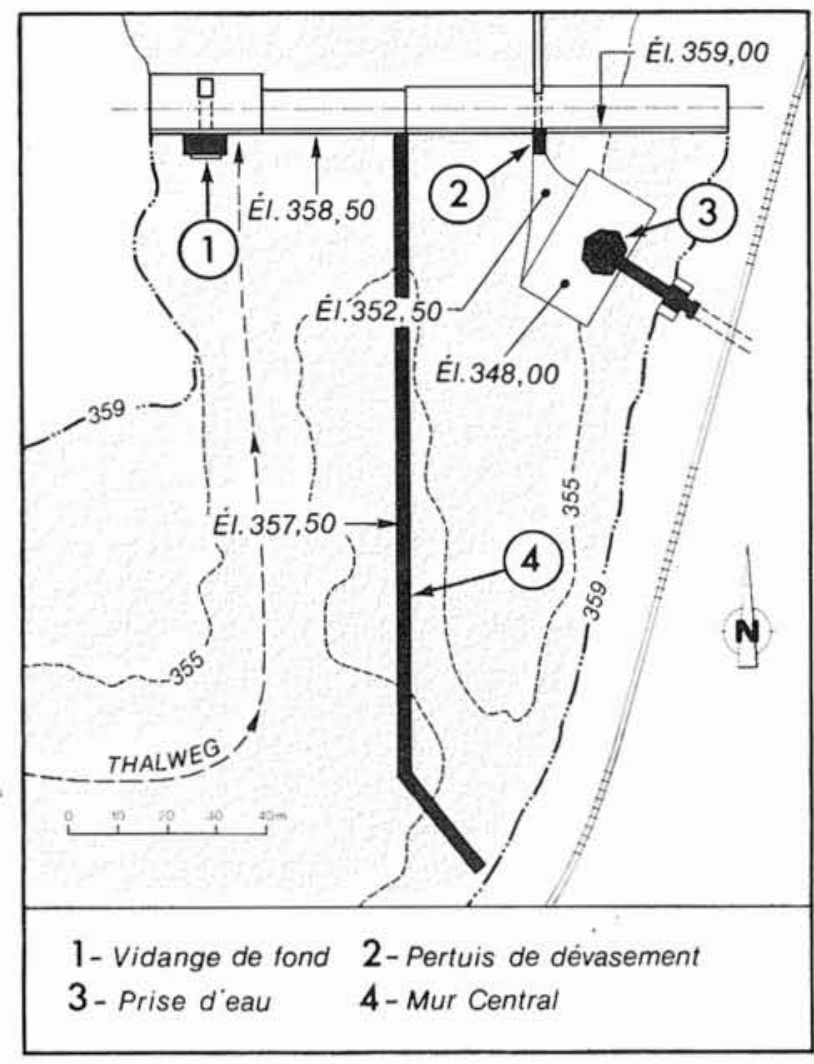

Figure 2. - Concept d'origine.

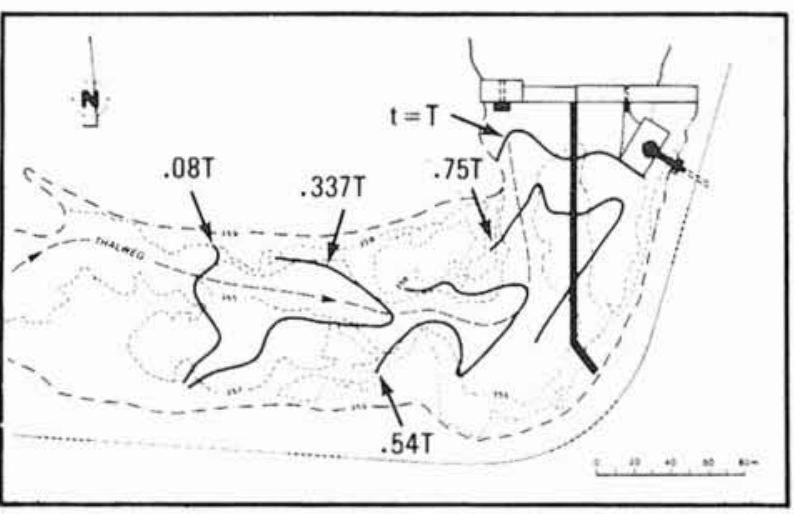

Figure 3. - Progression des dépôts vers la prise d'eau du concept d'origine.

\section{Différentes implantations étudiées}

L'implantation illustrée à la figure $4 a$ regroupait le concept de la prise en tube avec des vannes de grande capacité de part et d'autre de la rivière. Cette implantation possédait toutefois certaines caractéristiques indésirables de la disposition initiale, en particulier la concentration du débit sur la rive droite continuait à attirer l'eau au-dessus du mur, en ligne droite à partir du réservoir supérieur. En fonctionnement normal, alors qu'il n'y a pas de débit 


\section{Concept de barrage - prise d'eau sélectionnée}

Les essais systématiques décrits ci-dessus avaient démontré que l'implantation $4 \mathrm{~d}$ présentait des avantages distincts par rapport aux autres; cette configuration générale fut donc retenue pour des essais de mise au point.

Le modèle à échelle $1 / 50$ a été construit avec un fond fixe en ciment reproduisant les affleurements rocheux et les berges, y compris l'ouvrage du barrage et une partie du réservoir tel qu'illustré par la photo de la figure 5 .



Figure 5. - Photographie du modèle.
Les sédiments ont été simulés en utilisant du sable et de la sciure de bois de différentes grosseurs pour couvrir toute la gamme de matériaux prototypes de $0,2 \mathrm{~mm}$ à 150 $\mathrm{mm}$ de diamètre.

Une des caractéristiques importantes du réservoir réside dans le fait qu'il est divisé en biefs supérieur et inférieur par un coude à angle droit situé à $125 \mathrm{~m}$ en amont de l'axe du barrage. Dans tous les essais précédents, on a trouvé que le coude redirigeait très efficacement les sédiments pour que ceux-ci restent dans le chenal principal à l'approche du barrage.

Le programme d'essais détaillé a donc eu pour but de modifier les structures afin de tirer profit au maximum de ce mécanisme naturel de la rivière; l'implantation finale retenue est illustrée à la figure 6 . Une vue amont fait l'objet de la figure 7 avec vues en coupe aux figures 8 et 9 . Ce concept présente les éléments suivants :

- deux passes de chasse ou évacuateurs de crues, munies de vannes secteurs de $8 \mathrm{~m}$ de large dans le chenal profond adjacent au contrefort gauche, le seuil étant à l'élévation 351,0 .

- quatre ouvertures de prise d'eau munies de grilles à débris de $9 \mathrm{~m}$ de large chacune, allant de 353,85 à 356,50 d'élévation.

- un déversoir ayant une crête à l'élévation 359,0, divisé en huit passes avec une longueur totale de $80 \mathrm{~m}$.

- des dispositifs permettant l'ajout de batardeaux ou hausses, d'une hauteur de $1 \mathrm{~m}$, au déversoir.

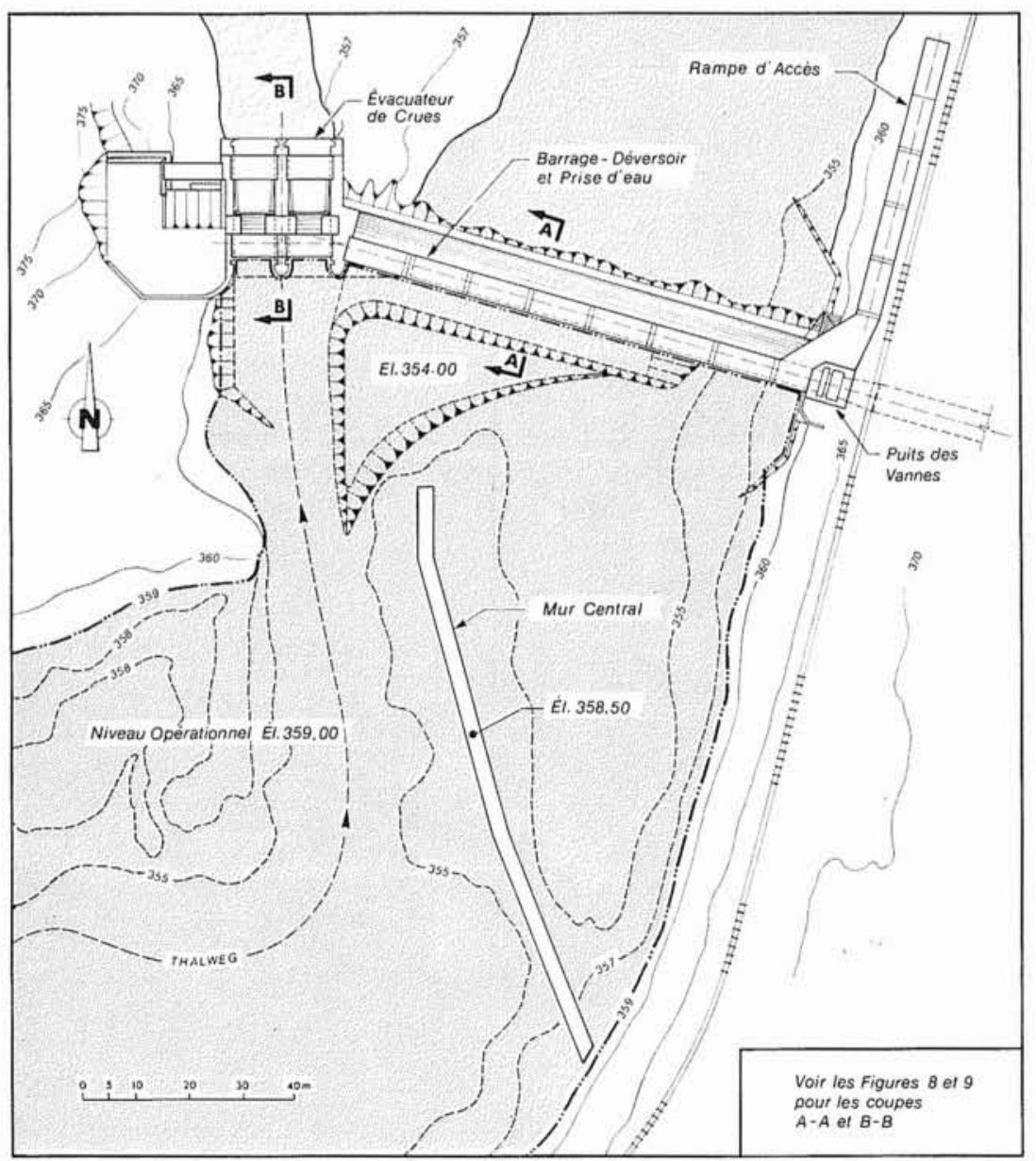

Figure 6. - Vue en plan de l'im. plantation modifiée. 


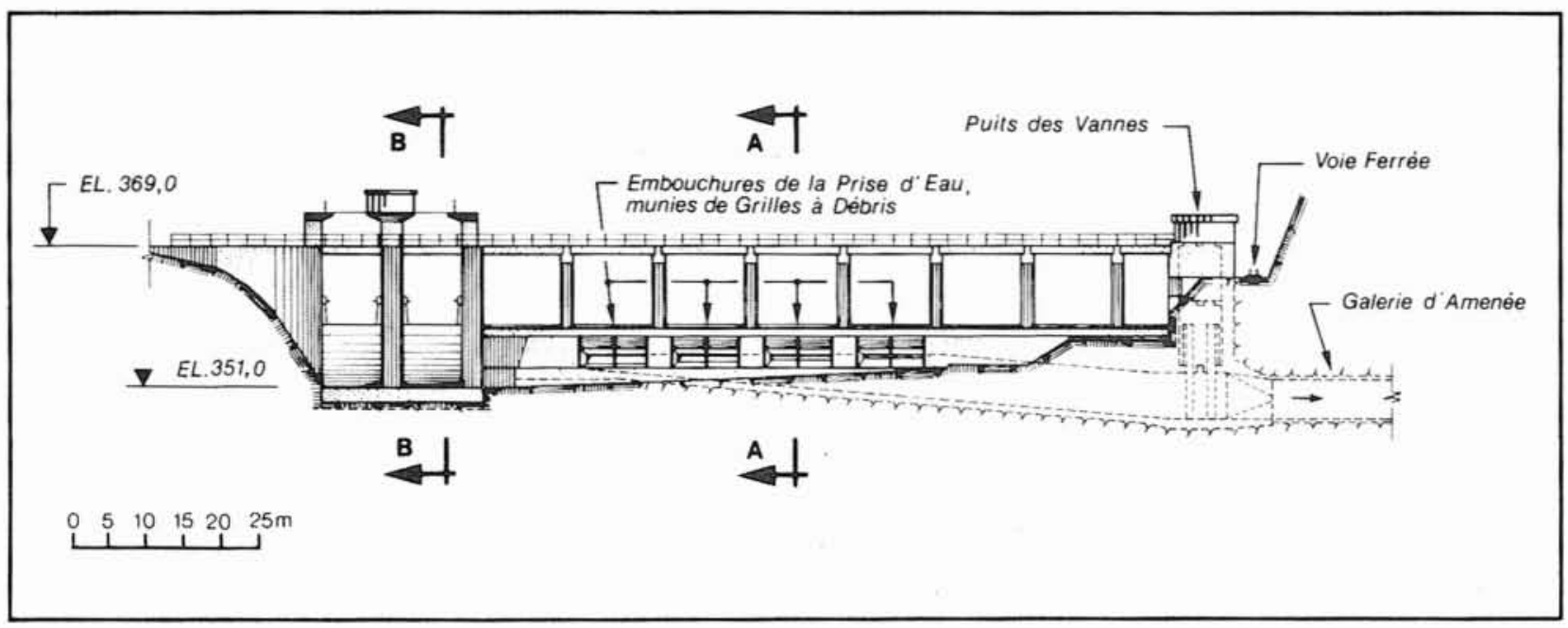

Figure 7. - Vue amont de l'implantation modifiée.

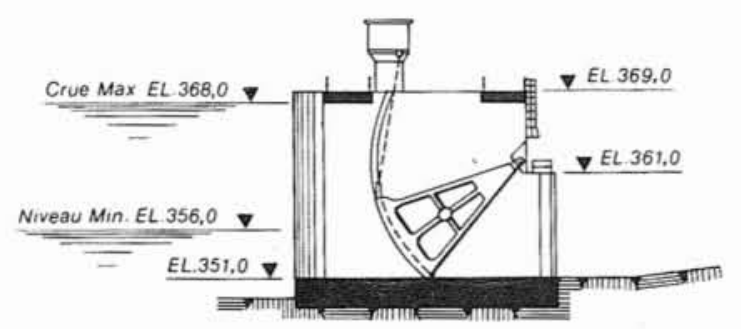

COUPE B-B EVACUATEUR DE CRUES

$$
\text { ㄴ. } 5 \text { : } 10 \text { : } 15 \text {. } 20 \mathrm{~m}
$$

Figure 8. - Coupe de l'évacuateur de crues - Implantation modifiée.

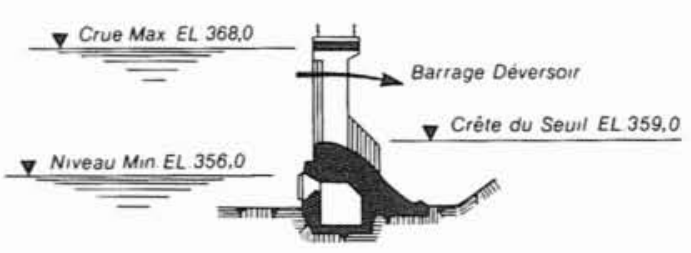

COUPE A-A PRISE D'EAU

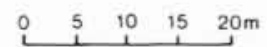

Figure 9. - Coupe du déversoir et de la prise d'eau - Implantation modifiée.

Figure 10. - Vue aval du barrage pendant la construction.

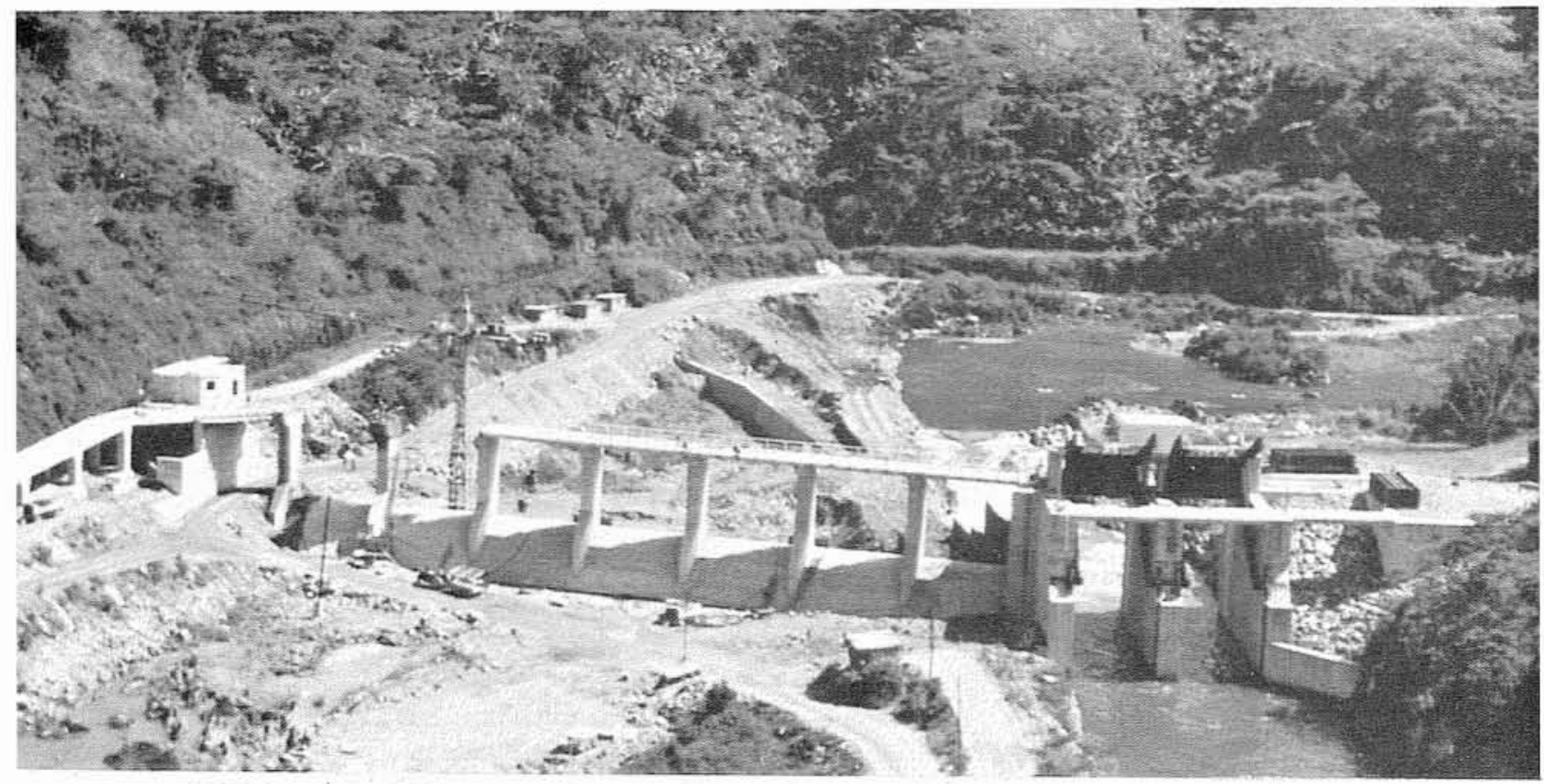


- un mur central submergé en amont avec crête à élévation 358,5 ,

- une rampe d'accès partant de la voie ferrée et arrivant à un pont au-dessus du déversoir, menant à l'ouvrage des passes à vannes-secteurs.

Des rainures de batardeaux ont été incorporées à l'ouvrage de l'évacuateur, en amont et en aval des vannes, avec un pont au-dessus (figure 10) à partir duquel une grue est utilisée pour déplacer les batardeaux. Etant donné qu'il n'y a pas de route dans la région, la grue est dotée de roues permettant ses déplacements routier et ferroviaire. Le débit de crues total qui pouvait être passé avec cette implantation s'est avéré légèrement plus élevé que pour le concept d'origine.

\section{Implantation modifiée Essais sur modèle}

Tout d'abord, des essais en eau claire ont été faits afin de déterminer les dimensions des vannes et du déversoir et les niveaux d'eau nécessaires pour passer la gamme complète de débits; plus spécifiquement, les inondations maximales posșibles pour que la voie ferrée ne soit pas inondée. De nombreuses observations furent effectuées utilisant des injections de colorant pour définir les courants secondaires engendrés par le coude à angle droit. Des essais ont été faits pour développer ces courants afin qu'ils transportent les sédiments vers les vannes secteurs.

Des essais successifs avec sédiments simulés permirent de mettre au point le niveau et la longueur du mur central, ainsi que la configuration des excavationsdevant les ouvertures de la prise. Le banc à l'élévation 354 sur la figure 6 fournit une zone d'écoulement additionnelle pour l'eau attirée par la prise, facilitant sa transition à partir du chenal principal. Au cours des périodes sans déversement, cette excavation accroît la partie du débit suivant le chenal principal par rapport à celle passant par-dessus le mur central.

En passant par le virage à angle droit où le chenal principal entre dans la retenue aval, l'écoulement génère un courant secondaire hélicoïdal dans le sens des aiguilles d'une montre. Ce courant, ou rouleau, persiste dans le trajet vers le barrage, entraînant les sédiments mobiles au fond vers la rive gauche, le long du thalweg.

Ainsi, un débit accru retenu dans le chenal principal y favorise la rétention des sédiments, soit sous forme de dépôt ou alors en prenant la direction des vannes. La figure 11 montre la configuration schématique de ce champ de vitesses.

Une fois le bout du mur central passé, le débit décrit un virage à droite vers les ouvertures de la prise d'eau. Un courant secondaire hélicoïdal dans le sens inverse des

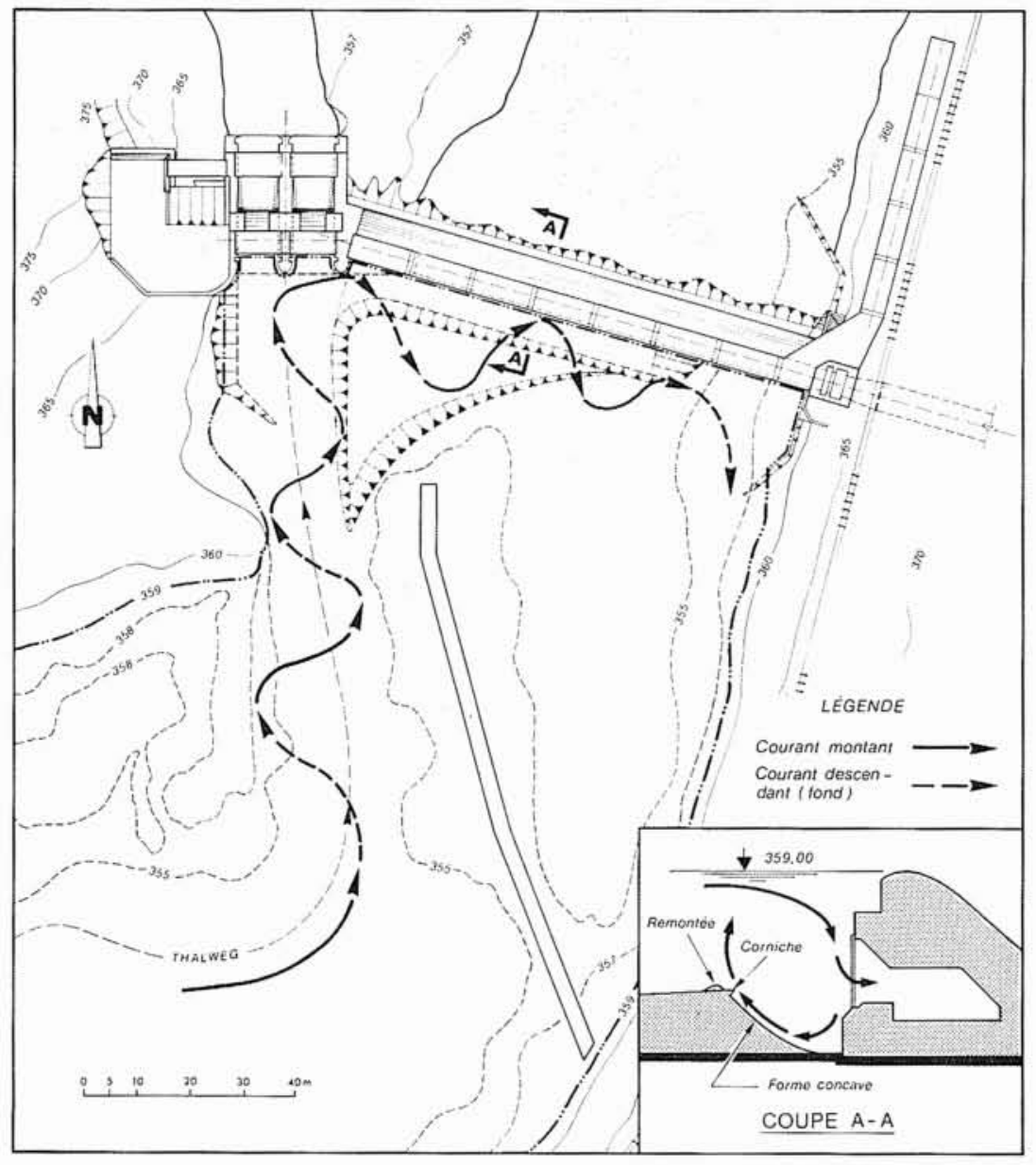

Figure 11. - Courants secondaires de la retenue. 


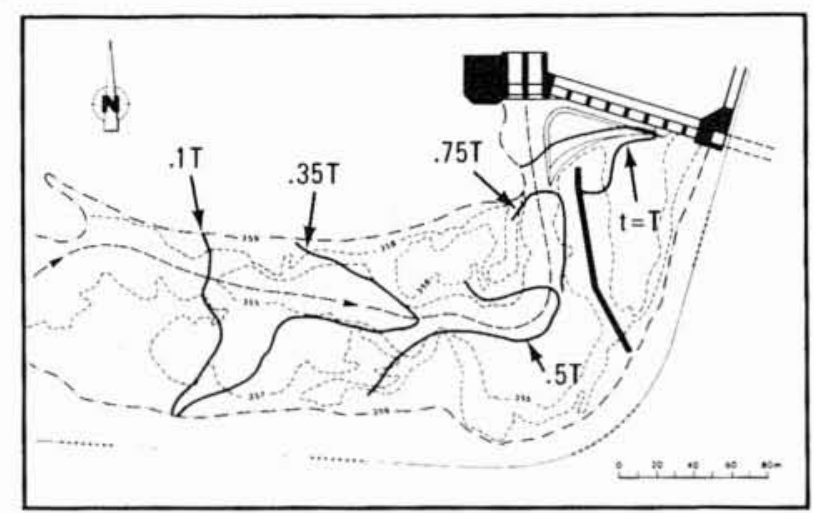

Figure 12. - Progression des dépóts vers la prise d'eau modifiée.

aiguilles d'une montre ou rouleau est ainsi créé devant le déversoir, aussi illustré à la figure 11 . Le chenal creusé le long de la face amont du déversoir a été dimensionné pour tirer le maximum de profit de cet effet de rouleau.

Tout sédiment ainsi rejeté de la prise d'eau sera déposé dans le bassin de prise et y demeurera jusqu'à ce que des crues de plus de $1500 \mathrm{~m}^{3} / \mathrm{sec}$. le portent au-dessus du déversoir.
Jusqu'à cette étape des études, les décisions-clés furent prises en tenant compte du cas de fonctionnement normal, avec tout le débit dérivé par la prise d'eau. Une série finale d'essais a été faite afin d'établir les données suivantes :

- relation niveau-débit pour la gamme complète de débits;

- champs de vitesses pour quatre niveaux de la retenue et trois débits;

- progression du débit de charriage à travers le réservoir.

Les résultats de ces derniers essais sont donnés à la figure 12 et on peut les comparer à ceux de la figure 3 . L'essai a été terminé lorsqu'il a été démontré que les matériaux de charriage atteignant la prise étaient soulevés et passaient par-dessus le déversoir en période de crue ou se trouvaient éloignés des grilles à débris par le rouleau, tel qu'illustré à la figure 11 . La figure 13 montre une vue du réservoir vide.

\section{Inspection du site - mai 1984}

La prise d'eau a été opérée suivant les consiğnes mises au point lors des essais sur modèle. Une inspection détaillée a été entreprise en 1984 afin d'évaluer l'état de la galerie non-revêtue et de déterminer si la prise d'eau s'était comportée tel que prévu. Avant l'assèchement de la galerie, une série d'observations furent faites dans la retenue afin de vérifier les prédictions du modèle concernant les rouleaux qui devraient exister dans le chenal principal et devant la prise d'eau alors que la centrale était en service.

Une solution concentrée de fluorescéine fut injectée sur le lit à un certain nombre de points dans la retenue et les trajectoires des courants de fond ont été déterminées par rapport à la direction des courants de surface. La figure 14 illustre les résultats de ces essais qui prouvent catégoriquement que le rouleau était bien formé et continu dans le chenal principal dirigé vers les deux passes de l'évacuateur de crues.

La présence du deuxième rouleau devant le déversoir n'a pu être matérialisée, vraisemblablement dû au fait que la centrale n'aspirait que $10 \mathrm{~m}^{3} / \mathrm{sec}$. alors que les vannes évacuaient plus de $100 \mathrm{~m}^{3} / \mathrm{sec}$. Cependant comme illustré par la $f i$ gure 15, photographie prise après la vidange de la retenue pour l'inspection, la prise d'eau est restée dégagée des dépôts fournissant la preuve indirecte de l'existence du rouleau protecteur. 
L'inspection de la galerie a montré que seules des quantités négligeables de sédiments s'étaient déposées. Il y avait une mince couche de sable sur la surface près de l'extrémité amont et les sédiments se limitaient au premier $100 \mathrm{~m}$. Un échantillon prélevé avait un $\mathrm{d}_{90}$ de $0,55 \mathrm{~mm}$, alors que plusieurs autres mesuraient $0,3 \mathrm{~mm}$, mais la majorité des matériaux était inférieure à $0,2 \mathrm{~mm}$ de diamètre. Les analyses de diffraction radiographiques ont indiqué que le sable contenait moins de $40 \%$ de quartz.

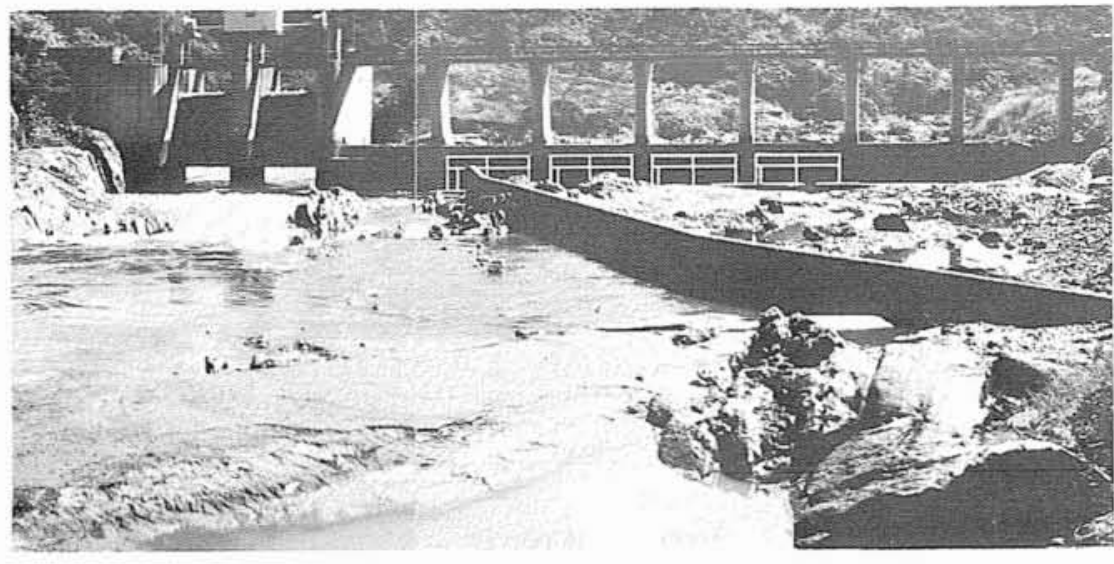

Figure 14. - Courants de surface et de fond dans la retenue.

\section{Conclusions}

Les études sur modèle réduit ont permis la mise au point d'une prise d'eau originale par tube-collecteur dans une rivière ayant un fort débit solide.

L'inspection de la prise d'eau et de la galerie d'amenée après deux ans de fonctionnement, a fourni une excellente occasion de vérifier le comportement de la prise. Cette inspection a révélé qu'elle fonctionne bien selon les prévisions du modèle. De plus, ces relevés ont indiqué que, pendant les périodes de crues, il y aurait une certaine quantité de solides aspirés dans la prise tel que prévu par l'étude sur modèle. Cependant, les quantités demeureront faibles, et la plupart des particules granulaires seront d'une grosseur inférieure à $0,2 \mathrm{~mm}$. Ainsi, le passage des matières solides à travers les turbines en acier inoxydable ne devrait causer que des dégradations minimes.

Les services d'experts-conseils ont été assurés par la société d'Ingénierie Cartier et les travaux sur modèle hydraulique ont été effectués par le Laboratoire d'Hydraulique LaSalle Ltée, situés tous deux à Montréal, Canada. La construction a été réalisée par Skanska de Suède.

\section{Remerciements}

Les auteurs désirent remercier Jirama, l'agence responsable de l'énergie et de l'approvisionnement en eau de la République Démocratique de Madagascar, qui a autorisé la publication de cette communication, ainsi que Skanska pour l'utilisation de la photographie de la figure 10. Ils remercient également M. S. Alam pour sa collaboration. Ce dernier était directement responsable des études sur modèle au Laboratoire d'Hydraulique La Salle.

Figure 15. - Chenal de la prise d'eau maintenu libre de dépôts sédimentaires par le rouleau - Retenue vide.

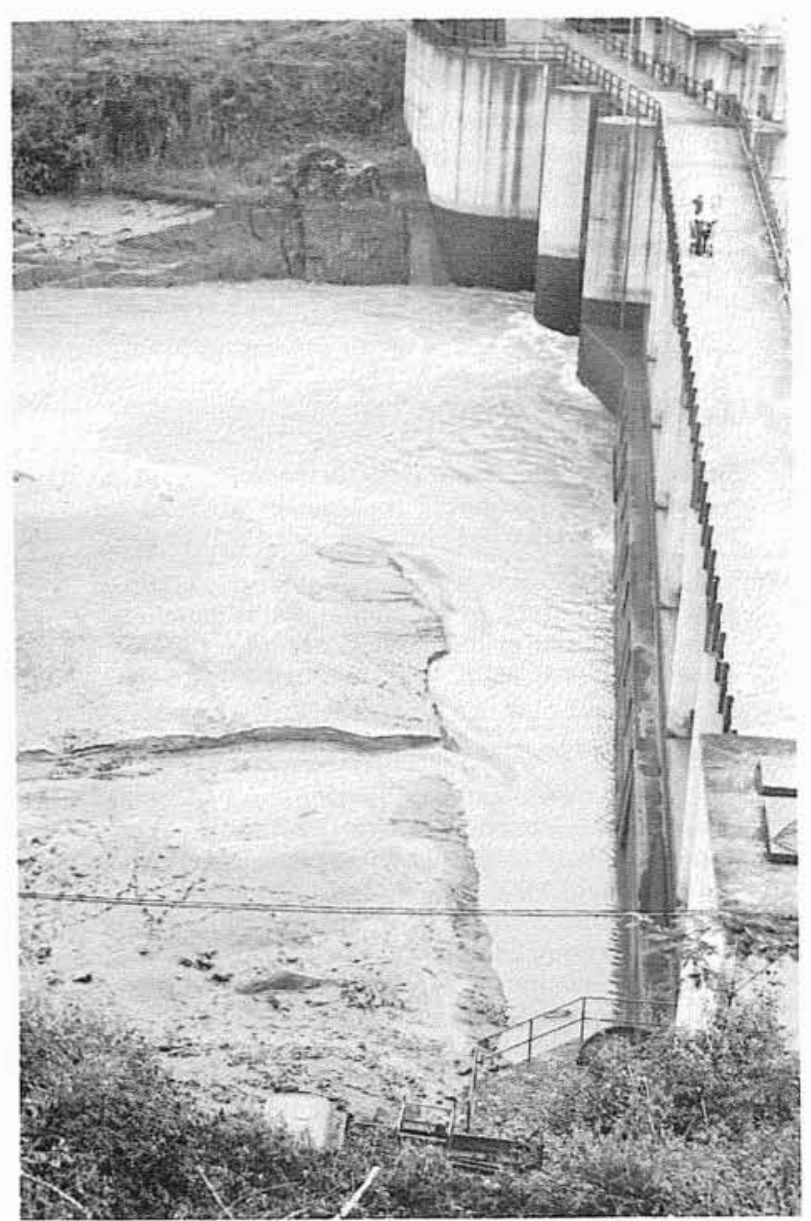

\section{Références}

[1] Randriamanantena C., Chartrand C., Aenishaenslin A. et VADIVEL K.P.A. - Projet Hydroélectrique Andekaleka, Comptes rendus de l'Association Electrique Canadienne (1984) Partie 3. 